\title{
A fully-autonomous hovercraft inspired by bees: wall following and speed control in straight and tapered corridors
}

\author{
Frédéric L. Roubieu, Julien Serres, Nicolas Franceschini, Franck Ruffier and Stéphane Viollet
}

\begin{abstract}
The small autonomous vehicles of the future will have to navigate close to obstacles in highly unpredictable environments. Risky tasks of this kind may require novel sensors and control methods that differ from conventional approaches. Recent ethological findings have shown that complex navigation tasks such as obstacle avoidance and speed control are performed by flying insects on the basis of optic flow (OF) cues, although insects' compound eyes have a very poor spatial resolution. The present paper deals with the implementation of an optic flow-based autopilot on a fully autonomous hovercraft. Tests were performed on this small (878-gram) innovative robotic platform in straight and tapered corridors lined with natural panoramas. A bilateral OF regulator controls the robot's forward speed (up to $0.8 \mathrm{~m} / \mathrm{s}$ ), while a unilateral OF regulator controls the robot's clearance from the two walls. A micro-gyrometer and a tiny magnetic compass ensure that the hovercraft travels forward in the corridor without yawing. The lateral OFs are measured by two minimalist eyes mounted sideways opposite to each other. For the first time, the hovercraft was found to be capable of adjusting both its forward speed and its clearance from the walls, in both straight and tapered corridors, without requiring any distance or speed measurements, that is, without any need for on-board rangefinders or tachometers.
\end{abstract}

\section{INTRODUCTION}

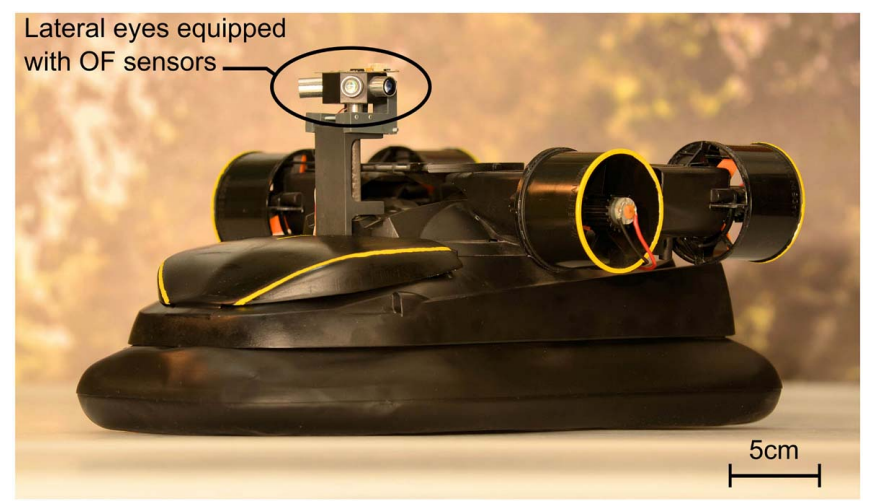

Fig. 1. Fully-autonomous sighted hovercraft equipped with miniature elementary eyes and a bio-inspired dual lateral optic flow regulator.

Winged insects, which emerged several hundreds of millions years ago, have developed elegant means of navigating in complex, highly unfamiliar environments. Their visually guided performances seem to depend mainly on Optic Flow (OF) cues, that is, on the angular speed $\omega$ (magnitude

Authors are with the Aix-Marseille University, CNRS, ISM UMR 7287, 13288, Marseille cedex 09, France ffrederic.roubieu, julien.serres, nicolas.franceschini,

franck.ruffier, stephane.viollet\}@univ-amu.fr in $\% / s$ ) at which any contrasting object moves past their eyes [1]-[4]. For example, insects have been found to use OF cues to control their lateral position in a corridor [3], [5] their ground height [6], [7], and their forward speed [8]-[10]. In insects' compound eyes, the OF is processed by neurons called Elementary Motion Detectors (EMDs), each of which is driven by photoreceptors with adjacent visual axes. Since OF sensors are non-contact, non-emissive - and therefore power-lean - visual sensors, they constitute invaluable tools for performing obstacle avoidance and speed control tasks, especially in the case of small autonomous robots, which have to meet particularly drastic energy and payload constraints. Behavioral studies on flying insects have provided researchers with innovative solutions for designing OF-based devices for the visual guidance of both ground vehicles [11]-[21] and aerial vehicles [18], [22]-[26]. Our previous simulation studies on the LORA III autopilot [20] were based on a dual lateral optic flow regulator controlling a simulated agent. The term optic flow regulator denotes a feedback loop which keeps the perceived OF at a constant value by adjusting one of the robot's thrusts [23]. The feedback sensor on which it is based is a 2-pixel OF sensor (called the Local Motion Sensor, - LMS -) based on a "time-of-travel" scheme [11], [27]-[31] which was originally inspired by the common housefly's EMD neurons [11], [12]. In Serres et al. [20] the simulated autopilot called LORA III, which was composed of a dual lateral optic flow regulator, was found to account not only for the typical centering behavior and speed control observed in honeybees flying in straight and tapered corridors [8], but also for the wallfollowing behavior that we recently described in honeybees [5]. Other robotic studies have dealt with OF-based guidance systems for underactuated and non-holonomic platforms, using either a rolling hovercraft [32]-[34] equipped with low friction rollerballs or a genuinely contactless hovercraft [35], [36] operating at a few millimeters above ground. Humbert et al. developed a simulated underactuated hovercraft [37] and an underactuated wheeled robot [21] equipped with a widefield camera, using a visual guidance system based on the spatial harmonic decomposition of the OF to estimate state parameters such as the forward speed and distance from obstacles. Zufferey et al. implemented an OF-based visual guidance system on a wheeled Khepera robot that showed wall-following behavior along a wall lined with a randomly generated highly contrasting pattern [38]: this sighted robot was equipped with a camera consisting of a 50-pixel 1-D array.

In the present study and for the first time, we implemented 
the LORA III dual lateral optic flow regulator onboard an original vehicle, that is, a miniature and fully actuated hovercraft (Fig. 1) equipped with two eyes mounted on opposite sides, each of which comprised only 2 pixels driving a single $\mathrm{OF}$ sensor, i.e. a single 2-pixel LMS. The hovercraft's visually-guided behavior was then tested in corridors lined with natural scenes. An additional headinglock feedback loop was also implemented to prevent any yaw rotations of the robot, and hence cancelling any rotational OF disturbances. This innovative robotic platform is presented in section II. In section III, the original implementation of the dual lateral optic flow regulator onboard the hovercraft is described in detail. Section IV deals with the control strategy used in the robotic platform and describes the dynamic identification of the robotic platform used to tune the hovercraft's controllers. In section V, the experimental set-up used to test the robot's behavior is presented. Section VI gives the experimental results obtained with the actual physical robot equipped with the LORA III autopilot.

\section{THE LORA HOVERCRAFT ROBOT}

\section{A. The robotic platform}

Our sighted LORA robot is a retro-fitted version of a miniature RC hovercraft (Taiyo Toy LtD, Typhoon T3) [35]. The miniature LORA robot (mass: $0.878 \mathrm{~kg}$, size: $0.36 \times 0.21 \times 0.17 \mathrm{~m}$, see Fig. 1) is fully actuated by means of four ducted fans (GWS EDF-50, DC motor CN12-RLC, mass: $30 \mathrm{~g}$ ) driving it on the horizontal plane. The two rear thrusters actuate the robot along the surge axis, the two lateral thrusters actuate the robot along the sway axis, and the robot's heading is adjusted by controlling the two rear thrusters differentially. An additional lift fan (a brushless motor Micro Rex 220/3-3200 Flyware fan, mass: 11g) inflates the skirt to create an air-cushion preventing the robot from touching the ground. The vehicle is naturally stable in terms of the pitch and roll and the very low friction coefficient and the number of degrees of freedom in the horizontal plane are similar to those of an aerial robot (a helicopter, for instance).

\section{B. The bio-inspired visual system}

Here we describe how the LORA robot navigates over a flat surface along a corridor (Fig. 5 and Fig. 4). The robot is equipped with four lateral eyes, i.e. four 2-pixel OF sensors placed at $\pm 45^{\circ}$ and $\pm 90^{\circ}$ azimuthal angles, as shown in Fig. 1. Only the two lateral eyes facing at azimuthal angles of $\pm 90^{\circ}$ are used here. To compensate for any yaw disturbances that would introduce an adverse rotational $\mathrm{OF}$ component into the angular speed measurements $\left(\omega^{m}\right)$, the robot is equipped with a custom-made heading-lock system composed of a micro-gyrometer (ADIS16100, range speed: $\pm 300^{\circ} / \mathrm{s}$, size $7 \times 7 \times 3 \mathrm{~mm}$, mass: $0.5 \mathrm{~g}$ ) and a micro-compass (HMC6052, precision: $0.3^{\circ}$, size: $24 \times 18 \mathrm{~mm}$, mass: $2 \mathrm{~g}$ ). The robot's heading $\Psi$, defined as the robot's yaw angle around the vertical axis, is therefore maintained along the $\mathrm{X}$-axis of the corridor $\left(\Psi=0^{\circ}\right)$. Each of the two lateral eyes therefore receives a purely translational OF (Fig. 5) $\omega_{R}$ and $\omega_{L}$ defined

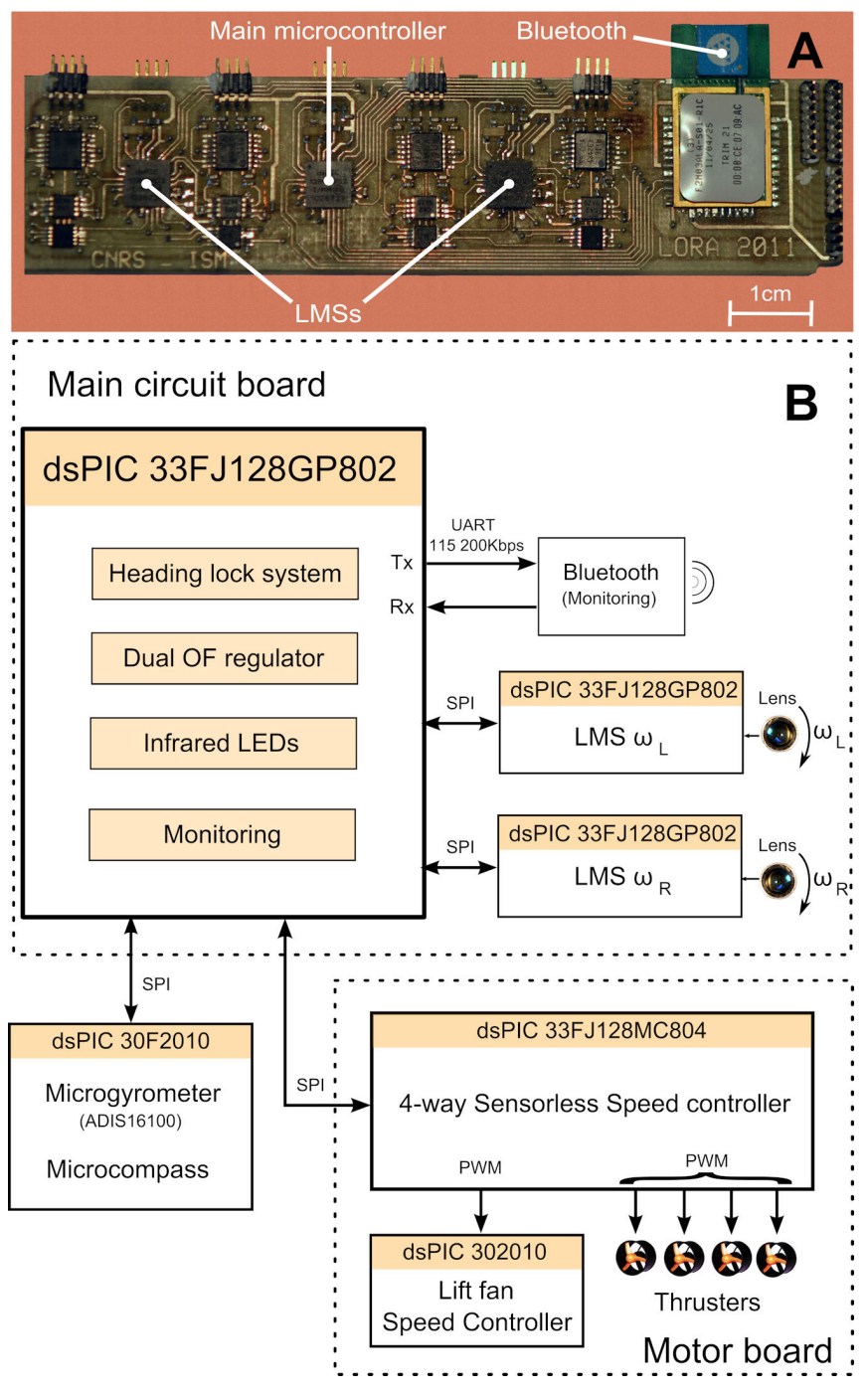

Fig. 2. Custom electronics: (A) Top view of the microcontroller-based $(\mu C)$ circuit board (size: 109x25mm, mass: $8 \mathrm{~g}$ ) including the embedded optic flow-based autopilot and visual LMSs. (B) Simplified scheme of the embedded electronics, showing the interconnections between the 6 tiny $\mu C s$.

as follows:

$$
\omega_{R, L}=\frac{V_{x}}{D_{R, L}}
$$

where $V_{x}$ is the robot's forward speed, $D_{R}$ and $D_{L}$ are the distances from the right and the left walls, respectively.

Each of the two OF sensors consists of an optical assembly composed of a lens and a pair of photosensors driving a single LMS running the "time of travel" scheme (see [11], [27]-[31] for further details). Each 2-pixel LMS measures the angular speed $\omega^{m}$, i.e. a 1-D component of the OF, generated by the robot's forward motion within a range of more than one decade $\left[25^{\circ} / s ; 350^{\circ} / s\right]$. Whenever an LMS does not detect any new contrasting features, it holds the last measured value for a period of $0.5 s$.

\section{Hardware architecture}

A photograph of the main electronic board is shown in Fig. 2A. The digital electronics embedded in this 
electronic board are the main microcontroller $(-\mu C$ dsPIC33FJ128GP802, clock: 40Mhz, working at a sampling frequency of $1 \mathrm{kHz}$ ) supervising two other dsPIC $\mu C s$ from Microchip(c) (dsPIC33F128GP802, clock: 40Mhz, working at a sampling frequency of $2 \mathrm{kHz}$ ) (Fig. 2B). The program of the main $\mu C$ was developed on Matlab/Simulink(c) and compiled using a specific toolbox (available on http://www.kerhuel.eu) dealing with multirate tasks such as: the heading-lock control system, the dual optic flow regulator, and the data monitoring during the main tasks (Fig. 2B). On the main electronic board, the two secondary $\mu C s$ (dsPIC33FJ128GP802) are in charge of the lateral OF processing (one $\mu C$ per eye). The main dsPIC therefore processes the visual inputs (i.e., the two OF measurements $\omega_{L}^{m}$ and $\omega_{R}^{m}$ ), the inertial input (i.e., the rate gyro $\dot{\Psi}^{m}$ ) and the vehicle's heading (i.e., the custom micro-magnetic compass $\left.\Psi^{m}\right)$. The Bluetooth device connected to the UART peripheral of the main $\mu C$ provides a full-duplex radio link between the robot and the $\mathrm{C} \sharp$-PC (C sharp) ground station. Via this radio link, data from the freely moving robot can be remotely logged and instructions such as the OF set-points, $\omega_{\text {SetFwd }}$ and $\omega_{\text {SetSide }}$, can be sent to the robot's autopilot, for example. Two separate Li-Polymer (LiPo) battery packs: a low-power pack (i.e., $7.2 \mathrm{~V}-360 \mathrm{mAh}$, size: $54 \times 31 \times 7 \mathrm{~mm}$, mass: $20 \mathrm{~g}$ ) for the electronics and a high-power pack (i.e., 7.2V-2200mAh, size: $21 \times 33 \times 96 \mathrm{~mm}$, mass: $112 \mathrm{~g}$ ) for the actuators give the robot an autonomy of 10 minutes. To prevent the rotor speed of the ducted fan to be affected by variations in the supply voltage, the first $\mu C$ (dsPIC33FJ128GP802, clock: 40Mhz) of the motor board controls each of the four propeller's rotational speeds (rpm) in a closed-loop mode on the basis of a dedicated "sensorless speed governor" [39]. This sensorless regulator makes each ducted fan rpm robust to large voltage disturbances while preserving the short rise time. The second $\mu C$ in the motor board controls the lift fan rpm in a closed loop mode via an optical sensor. Making the robot robust to voltage disturbances was a prerequisite for identifying the system before closing the visuo-motor loops. The LORA robot moves freely without any umbilical links. It is fully autonomous as it performs all the processing steps onboard and carries all its sensors and actuators, its own wireless data link and its own power supplies. To summarize, the 6 microcontrollers distributed in the LORA robot handle no less than 8 feedback-loops and make the robot relatively immune to heading disturbances and power supply variations.

\section{DUAL LATERAL OPTIC FLOW REGULATION}

A slightly upgraded version of the original LORA III autopilot [20] was implemented onboard the current LORA robot. In addition to the dual lateral OF regulator, another feedback loop was introduced to make the robot move straight along the corridor axis. The autopilot therefore controls the robot's motion not only forward and sideways but also about the yaw axis, according to the following principles:

- the heading feedback loop, composed of an inner- and an outer-feedback loop, stabilizes the robot's yaw angle at $\Psi=0^{\circ}$ to cancel any yaw drift. It therefore keeps the robot aligned with the corridor's main axis thanks to a custom-made magnetic micro-compass enhanced by a tiny micro-rate gyro;

- the first $O F$ regulator is a unilateral $O F$ regulator that adjusts the vehicle's lateral thrust (which determines the sway speed $V_{y}$ ) so as to keep the higher of the two lateral OFs perceived, that is, $\max \left(\omega_{R, L}^{m}\right)$, equal to the sideways OF set-point $\omega_{\text {SetSide. }}$. The distance from one wall thus becomes proportional to the robot's forward speed $V_{x}$ (as defined in the section $2 \mathrm{~B}$ ). The faster the robot travels, the further away from that wall it will therefore be. The clearance from the walls depends mainly on the sideways $O F$ set-point;

- the second $O F$ regulator is a bilateral $O F$ regulator that adjusts the robot's forward thrust (which determines the surge speed $V_{x}$ ) so as to maintain the sum of the two (right and left) OFs, that is, $\Sigma \omega_{R, L}^{m}$, equal to the forward OF set-point $\omega_{\text {SetFwd }}$. The robot's forward speed therefore automatically adjusts itself proportionally to the local corridor width $D$, although the latter is not specified explicitly. The forward speed attained by the LORA robot will depend mainly on the forward $O F$ set-point.

All three feedback loops are implemented in parallel on the $\mu C$ of the main circuit board. Once the OF-based autopilot has reached the steady state, the LORA robot will travel at a given forward speed $V_{x}$ and at a given distance from one wall $\left(D_{L}\right.$ or $\left.D_{R}\right)$. The steady state operating point of the LORA robot $\left(V_{x \infty}, D_{R, L \infty}\right)$ is defined in [20].

\section{DYNAMIC MODEL AND CONTROL OF THE HOVERCRAFT}

Each of the four thrusters equipping the LORA robot is composed of a DC motor loaded with a light three-blade propeller producing a thrust of up to $0.72 \mathrm{~N}$ at $7.2 \mathrm{~V}$, in proportion to the voltage applied [36]. Since the propeller's profile is not symmetrical and reversing the thrust would therefore not brake the robot, the lift-fan was used to smoothly deflate the skirt, as suggested in [36], and thus served as a "brake". This active braking is achieved by combining the control signals of the rear thrusters and those of the lift fan $u_{\text {LiftFan }}$ in a single controller (the forward controller $C_{F w d}(s)$ ). Any decrease in the lift fan control signal $u_{\text {LiftFan }}$ will increase the robot's forward and side linear viscous friction coefficients $\left(\zeta_{F w d}\right.$ and $\zeta_{\text {Side }}$ respectively with $\zeta_{F w d, \text { Side }}=\frac{m}{\tau_{F w d \text {,Side }}}$ ), and thus decrease both the forward and side time constants $\tau_{F w d}$ and $\tau_{\text {Side }}$. We therefore identified the forward and side dynamics of the hovercraft $\left(G_{F w d}(s)\right.$ and $G_{\text {Side }}(s)$ respectively) corresponding to several lift fan output signals $u_{\text {LiftFan }}$. In the lift fan control signal range of $[37 \% ; 42 \%]$, the forward and side dynamic models of our hovercraft can therefore be described as a first-order low-pass filter with a time constant $\tau_{F w d, \text { Side }}$, but the side dynamic model of the robot also requires an integrator since the output is a position. The yaw dynamic model $G_{\Psi}(s)$ can be described as a low-pass filter also incorporating an integrator 


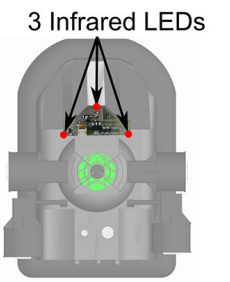

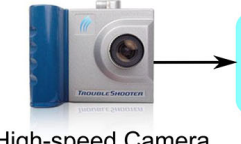

High-speed Camera $125 \mathrm{fps}$
High-resolution grayscale image $1280 \times 512$ pixels

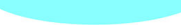

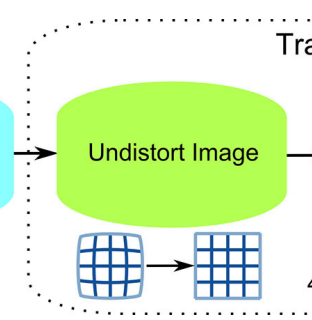

Tracking algorithm (offline)

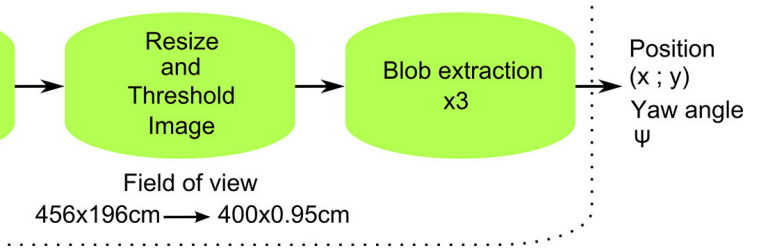

Fig. 3. Block diagram showing the image processing performed on the robot's trajectories along a corridor. A wide-angle lens (Pentax B618DX, focal length: $6.5 \mathrm{~mm}$, Field of View - FOV - : $97.91 \times 76.28^{\circ}$ ) was used to film the 4-meter long corridor. The camera FOV was adjusted to $456 \mathrm{~cm}$ in length and $196 \mathrm{~cm}$ in width, and centered on the corridor, to film the whole scene. Each trajectory recorded was saved on a computer via Midas $(4) 4.0$ Express (for Fastec cameras) and processed offline on a computer equipped with Matlab (c) R2008b for image processing. The robot's trajectories were recorded by adding an infrared filter (wavelength $\geqslant 850 \mathrm{~nm}$ ) to the camera. Three infrared photodiodes (SFH4050, half angle $\pm 80^{\circ}$ ) were mounted at triangular points on the robot so that only LED blobs showed up on the image. The robot's trajectory was reconstructed with a custom-made Matlab@ algorithm that analyzed sequential video frames offline. This algorithm first corrected the barrel distortion induced by the wide-angle lens via a camera calibration procedure, using "Camera Calibration Toolbox" (www.vision.caltech.edu/bouguetj/calib_doc) for Matlab@). The image was then resized and thresholded so that only the blobs corresponding to the infrared LEDs showed up, and each blob position was recorded. The LORA robot's position was measured from the barycenter of the three blobs, whereas the orientation of the LED triangle gave the robot's orientation with respect to the X-axis of the corridor.
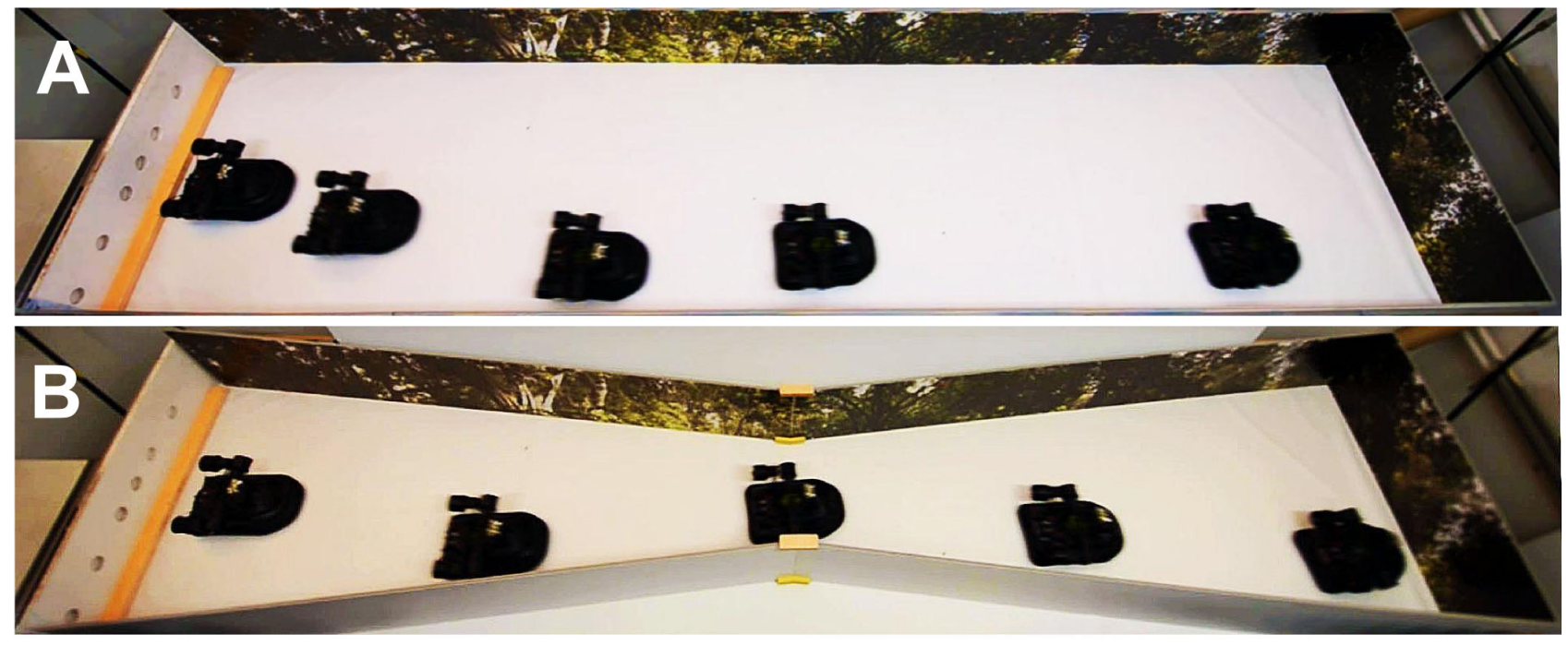

Fig. 4. Chronophotography of the LORA robot equipped with its OF-based autopilot (see Section III) in (A) a straight (1.12-second time interval) and (B) a tapered corridor (1.83-second time interval) lined with natural colored scenes. The autonomous fully actuated LORA robot entered the corridor without being informed about the width or configuration of the corridor and therefore operated in (A) and (B) a wall-following behavior.

(see Tab. I). Static gain and time constant parameters of the identified forward and side dynamics corresponding to each control signal $u_{\text {LiftFan }}$ are given in Tables II and III, respectively. Data of the robot's identification show that the surge and sway time constants ( $\tau_{F w d}$ and $\tau_{\text {Side }}$ respectively) are on the same order of magnitude because the air cushion of the hovercraft is homogeneous at any point under the robot, involving the forward and side linear viscous friction coefficients $\zeta_{F w d \text {,Side }}$ to be therefore on the same order of magnitude at any point under the air cushion.

Dynamic identification of the hovercraft enabled us to tune the appropriate controllers for the heading feedback loop and the OF-based autopilot's two visuo-motor feedback loops [20]. A proportional-integral (PI) controller $C_{F w d}(s)$ was introduced into the forward feedback loop to improve the closed-loop dynamics and obtain a zero steady-state error. The error signal $\varepsilon_{F w d}$ feeding the forward controller is calculated as follows:

$$
\varepsilon_{F w d}=\omega_{S e t F w d}-\Sigma \omega_{R, L}^{m}
$$

According to the sign of the output control signal of the forward controller, the robot can therefore:

- accelerate by controlling the rear thrusters in the ]0;60\%] range (Pulse Width Modulation control signal) and $u_{\text {LiftFan }}$ to its maximum value of $42 \%$;

- "brake" by switching off the rear thruster and by decreasing $u_{\text {LiftFan }}$ in the identified range of $[37 \% ; 42 \%]$.

A lead integrator $C_{\text {Side }}(s)$ was introduced into the side feedback loop to increase the damping, thus improving the stability and the sway dynamics. The error signal $\varepsilon_{\text {Side }}$ is calculated as follows:

$$
\varepsilon_{\text {Side }}=\omega_{\text {SetSide }}-\max \left(\omega_{R, L}^{m}\right)
$$

Only one lateral thruster is actuated at a time according to the sign of the output control signal of the side controller: 


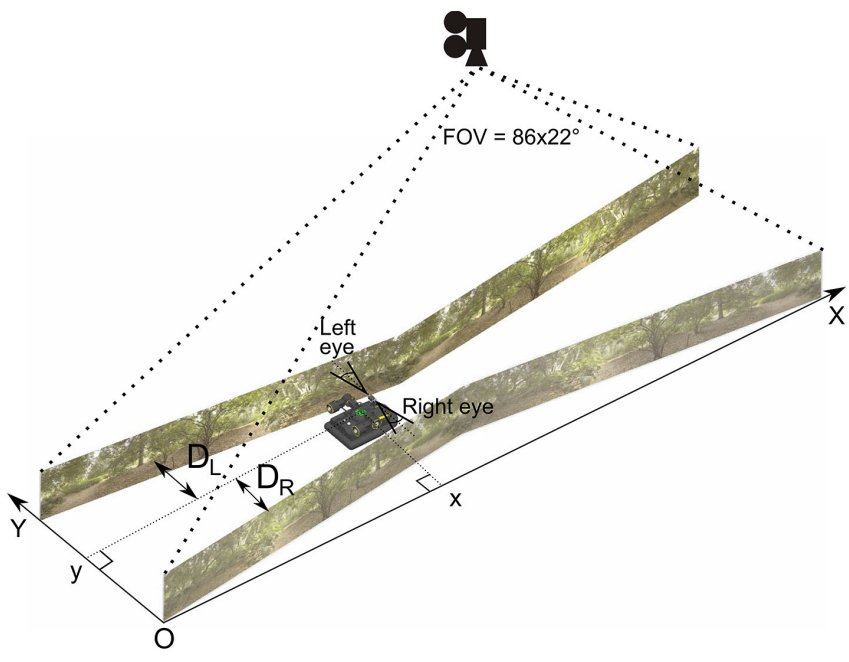

Fig. 5. The tapered corridor consisted of a 400-cm long corridor with a relatively wide entrance $(95 \mathrm{~cm})$ and with a $46-\mathrm{cm}$ wide constriction located midway, the lateral walls of which were lined with photographs of natural colored scenes (trees and bushes). A high-speed camera equipped with a wide-angle lens (angle of view of the corridor imaged by the camera: $86 \times$ $22^{\circ}$ ) placed $209 \mathrm{~cm}$ above the corridor filmed the whole scene at a rate of $125 \mathrm{fps}$. The high-speed camera was carefully calibrated and equipped with an infrared filter to record the trajectory of the robot via 3 infrared LEDs mounted at triangular points at the top of the robot.

- the left thruster is actuated and the right one is off;

- the right thruster is actuated and the left one is off.

As regards the heading-lock system, a proportional controller $H_{c}$ was included in the outer feedback loop (based on the magnetic micro-compass) and a PI controller in the inner feedback loop (based on the micro-gyrometer) to improve the closed-loop dynamics and obtain a zero steady-state error, thus locking the robot's heading to the X-axis of the corridor (see Fig. 5 and Fig. 4). All the transfer functions presented in this section are summarized in Table I. The vision-based autopilot controls the LORA robot at a sampling frequency of $100 \mathrm{~Hz}$.

\section{EXPERIMENTAL SET-UP}

To assess the performances of the LORA robot, we built two types of environment: a straight, 0.95-meter wide, 4meter long corridor (Fig. 4A), and a tapered 4-meter long corridor (tapering angle: $7^{\circ}$ ) (Fig. 4B) with a 0.95 -meter wide entrance and a 0.46-meter wide constriction located midway. All the corridor walls were lined with natural colored scenes showing trees and bushes, the rich texture of which favored a high refresh rate in the OF sensors' measurements [30]. The experiments were carried out indoors under artificial lighting conditions. A high-speed (125 fps), high-definition (1280 x 512 pixels) digital camera (a Fastec Imaging TroubleShooter camera) and equipped with an infrared filter was placed on a rigid mount $2.09 \mathrm{~m}$ above the ground (Fig. 5) and was used to record the robot's trajectories (Fig. 3).

\section{EXPERIMENTAL RESULTS}

All the results obtained in this study correspond to tests performed on the actual physical robot LORA. It is worth
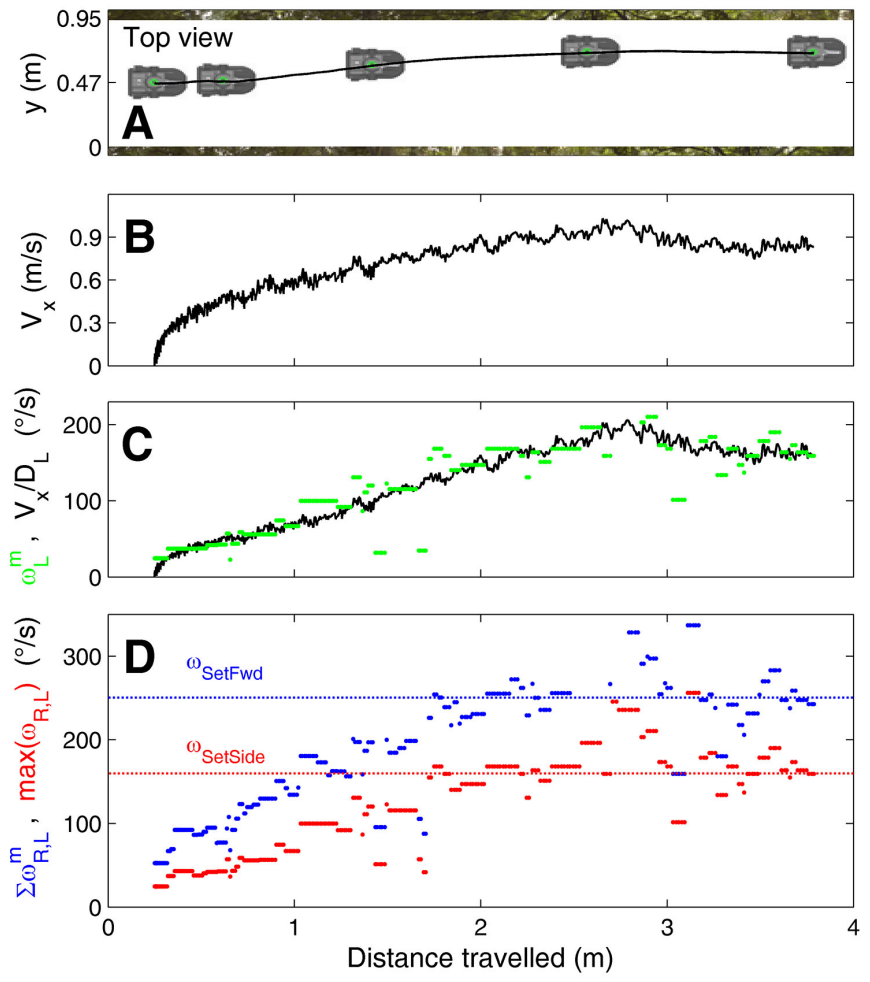

Fig. 6. Detailed example of the automatic wall-following behavior observed with the autopilot based on the dual optic flow regulation. The LORA robot started at the center of a straight corridor with $\omega_{\text {SetFwd }}=250^{\circ} / \mathrm{s}$ and $\omega_{\text {SetSide }}=160^{\circ} / \mathrm{s}$ OF set-points. (A) Recording of the robot's trajectory in the horizontal plane (Marks on the trajectory indicate the robot's position every 1.6 seconds). (B) Forward speed profile $V_{x}$ corresponding to the trajectory shown in (A). (C) Superimposition of the actual OF generated by the robot's own motion, as computed from equation 1 (black solid line) and the OF measured by the left LMS $\omega_{L}^{m}$ (green dash-dotted line). The quality of the LMS output signal $\omega_{L}^{m}$ was virtually the same as the actual OF computed in (C). (D) The bilateral OF regulator strives to maintain the sum of the measured lateral OF $\Sigma \omega_{R, L}^{m}$ (red dash-dotted line) equal to the forward OF set-point $\omega_{\text {SetFwd }}$ (red dotted line). Jointly to the bilateral OF regulator, the unilateral $O F$ regulator strives to maintain the higher of the maximum lateral $\mathrm{OF}$ measurement $\max \left(\omega_{R . L}^{m}\right)$ (red dash-dotted line) to the sideways OF set-point $\omega_{\text {SetSide }}$ (red dotted line). In the steady state, which was reached only in the second half of the corridor, the robot attained a forward speed $V_{x \infty}=0.9 \mathrm{~m} / \mathrm{s}$ while maintaining a constant clearance $D_{L_{\infty}}=30 \mathrm{~cm}$ from the left wall (wall-following behavior).

mentioning that the LORA robot is never provided with any explicit information about either its current forward speed $V_{x}$, its current distance from the walls $D_{R}$ or $D_{L}$, the local corridor width $D$ or any informations about the corridor configuration. Fig. 6 shows the trajectory of the LORA robot gliding safely along a straight corridor, with the following set-points: $\omega_{S e t F w d}=250^{\circ} / \mathrm{s}$ and $\omega_{\text {SetSide }}=160^{\circ} / \mathrm{s}$. Marks on the trajectory (Fig. 6A) show that the robot automatically accelerated and stabilized its forward speed in the steady state, which was reached only in the second half of the corridor as shown in Fig. 6B. The sideways OF regulator strived to maintain the maximum measured lateral $\mathrm{OF}$, that is, $\max \left(\omega_{R, L}^{m}\right)$ (Fig. 6D, red dash-dotted line), at a constant value corresponding to the sideways OF set-point $\omega_{\text {Side }}$ and jointly the forward OF regulator strived to maintain the sum of the measured lateral OF, that is, $\Sigma \omega_{R, L}^{m}$ (Fig. 6D, blue dash- 

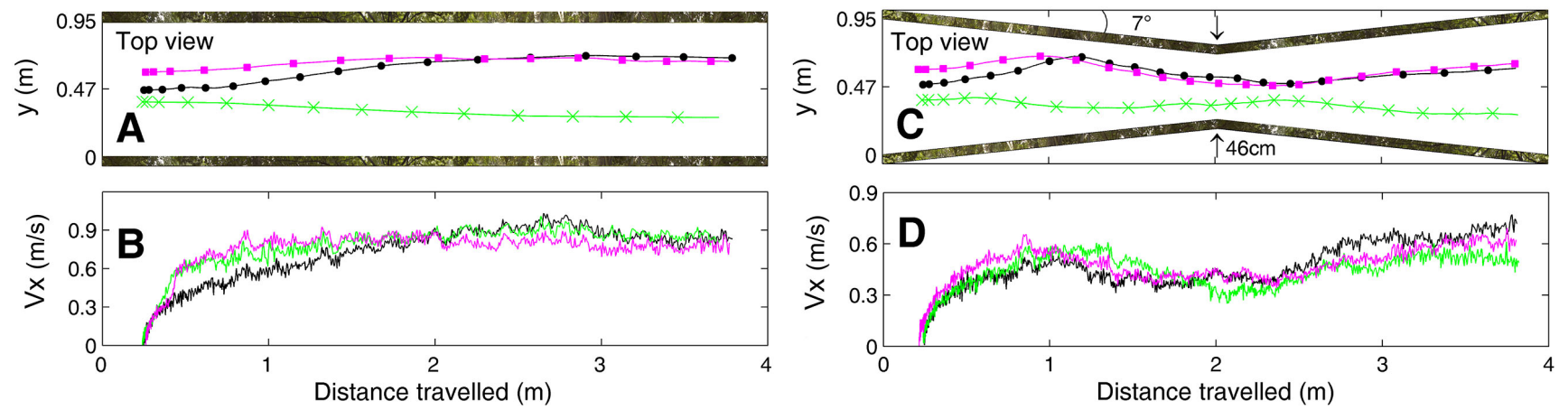

Fig. 7. Automatically safe wall-following behavior in both straight and tapered corridors with no informations supplied about the corridor configuration (corridor width $D$ or tapering angle for instance). This behavior occurs whenever $\omega_{\text {SetSide }}>\frac{\omega_{\text {Set Fwd }}}{2}$. The forward OF set-point was $\omega_{\text {SetFwd }}=250^{\circ} / s$ and the sideways OF set-point was $\omega_{\text {SetSide }}=160^{\circ} / \mathrm{s}$ (Marks on trajectories indicate the robot's position every 0.4 seconds). (A) Actual wall-following trajectories of the LORA robot in a straight corridor with three different starting positions. (B) Forward speed profiles corresponding to the trajectories shown in (A). In all three cases, the forward speed reached $0.9 \mathrm{~m} / \mathrm{s}$ in the steady state, with a clearance of $0.31 \mathrm{~m}$ from one wall. (C) Actual wall-following trajectories of the LORA robot in a tapered corridor (tapering angle: $7^{\circ}$ ) with three starting positions. (D) Forward speed profiles corresponding to the trajectories shown in (C). Results show the LORA robot automatically adjusts its speed with the corridor width while preserving a safe clearance to one wall.
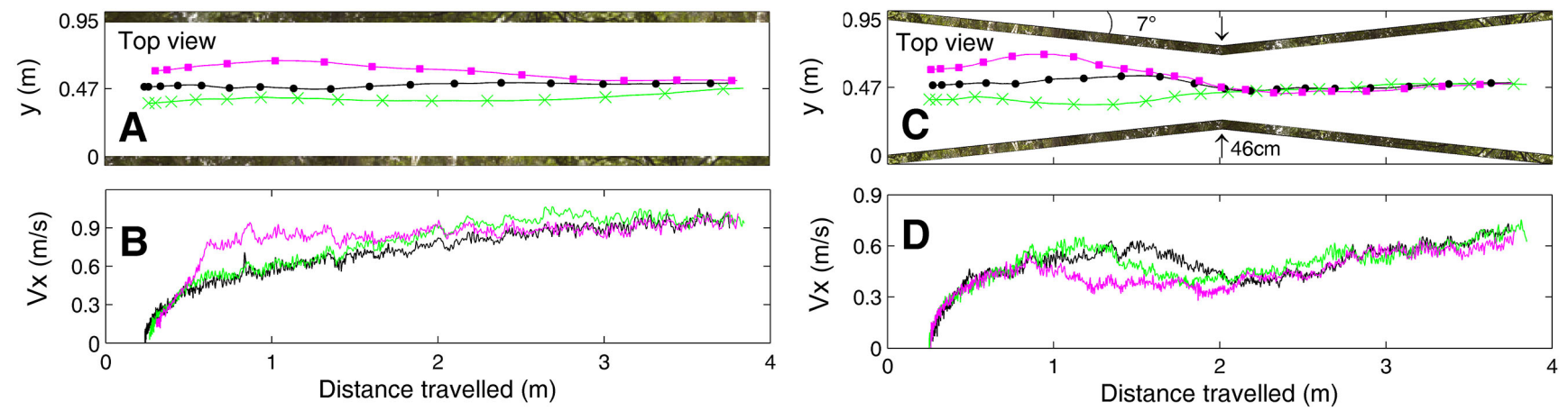

Fig. 8. Automatic centering behavior in both straight and tapered corridors with no information about the tapering angle. This is a particular case of the wall-following behavior, which occurs whenever $\omega_{\text {SetSide }} \leq \omega_{\text {SetFwd }} / 2$. The forward OF set-point here was $\omega_{\text {SetFwd }}=250^{\circ} / \mathrm{s}$ and the sideways OF set-point was $\omega_{\text {SetSide }}=125^{\circ} / \mathrm{s}$ (Marks on the trajectories indicate the robot's position every 0.4 seconds). (A) Actual centering trajectories taken by the LORA robot along a straight corridor with various staring positions. (B) Forward speed profiles corresponding to the trajectories shown in (A). In the steady state, the forward speed reached $0.9 \mathrm{~m} / \mathrm{s}$ in all three cases. (C) Actual centering trajectories taken by the LORA robot along a tapered corridor (tapering angle: $7^{\circ}$ ) with various starting positions. (D) Forward speed profiles corresponding to the trajectories shown in (C). The trajectories recorded in both the straight and tapered corridors show that in the steady state, the LORA robot automatically adjusted its forward speed and navigated safely along the centre of the corridor (centering behavior).

dotted line), to the forward set-point $\omega_{S e t F w d}$. This made the LORA robot glide safely at a constant clearance $D_{L}$ from the left wall and at a constant speed $V_{x}$ in the second half of the corridor. In Fig. 6C, one of the two output signals $\left(\omega_{L}^{m}\right.$, green dotted line) feeding the autopilot was virtually the same as that of the actual OF value $\left(\frac{V_{x}}{D_{L}}\right.$, black solid line $)$ computed from equation 1 , which confirms the accuracy of the OF measurements. Figures 7 show real trajectories along a straight (Fig. 7A) and a tapered corridor (Fig. 7C) (tapering angle: $7^{\circ}$ ) with $\omega_{\text {SetFwd }}=250^{\circ} / \mathrm{s}$ and $\omega_{\text {SetSide }}=160^{\circ} / \mathrm{s}$ setpoints. The LORA robot navigated safely and followed one of the two walls, regardless of its initial position $y_{0}$ at the entrance to the corridor. Whether in a straight (Fig. 7A) or tapered (Fig. 7C) corridor, the robot ended up by following either the right or the left wall in the steady state, depending on its initial ordinate $y_{0}$. These trajectories are typical of the wall-following behavior observed. The robot's speed profiles in the straight and tapered corridors (Figs. 7B and 7D) show that the LORA robot consistently adjusted its forward speed $V_{x}$ to the local corridor width $D$. The LORA robot typically slowed down when the local corridor width decreased and speeded up when it widened out after the constriction. Fig. 8 shows real trajectories along a straight corridor (Fig. 8A) and a tapered corridor (Fig. 8C) (tapering angle: $7^{\circ}$ ) with $\omega_{\text {SetF } w d}=250^{\circ} / \mathrm{s}$ and $\omega_{\text {SetSide }}=125^{\circ} / \mathrm{s}$ setpoints. The LORA robot navigated safely regardless of its initial position $y_{0}$ at the entrance to the corridor. In both cases, the robot ended up by following the right and left walls alternately: all these trajectories typical of the centering behavior were exactly as expected in [5]. The forward speed $V_{x}$ (Figs. 8B and 8D) can be seen to have been proportional to the local corridor width $D$, as occurred with both our simulated LORA robot [20] and honeybees [8].

\section{CONCLUSION AND FUTURE WORKS}

Insect-inspired visuo-motor control systems can suggest robotic solutions requiring a much fewer pixels than the present-day mobile robots harnessed to computer-vision systems. Here we describe the physical implementation of an 
elementary insect-inspired autopilot on a miniature fully actuated hovercraft (mass: $878 \mathrm{~g}$, size: $0.36 \times 0.21 \times 0.17 \mathrm{~m}$ ). This autopilot was based on the dual lateral optic flow regulation principle. The results obtained confirm the validity of our previous computer-simulated experiments [20]. The autopilot causes the robot to automatically adjust its forward speed $V_{x}$ to the local width $D$ of the corridor, while ensuring a safe clearance $\left(D_{R}\right.$ or $\left.D_{L}\right)$ from the walls, in line with honeybees' behavior in similar situations [3], [5], [8]. As long as the surrounding walls are textured, LORA's minimalist visual system, comprising only 4 pixels, enables the robot to navigate safely along an unknown straight or tapered corridor without being informed about its own speed, its distance from the walls, the corridor's width, or the tapering angle in the case of the tapered corridor (see Section VI). This visionbased autopilot therefore needs to measure (or estimate) neither the robot's speed (contrary to [21], [24], [25], [34], [37]) nor its clearance from the walls (contrary to [21], [34], [37]). An autopilot of this kind obviously generates major savings in terms of the onboard avionics and data processing requirements, which may explain why this OF-based guidance system matches insects' limited processing resources. In addition, the OF-based autopilot presented in this study enables the LORA robot to autonomously perform various tasks such as wall-following (Fig. 7) and centering (Fig. 8) without having to switch abruptly from one strategy to another. Whether the robot follows a wall or centers depends purely on the values of the two OF set-points $\omega_{\text {SetFwd }}$ and $\omega_{\text {SetSide }}$. The results obtained here show that a fully actuated ground vehicle equipped with an autopilot based on the dual lateral optic flow regulation principle may explain the result of recent behavioral experiments showing that honeybees do not necessarily center when travelling along a corridor [5]. The honeybee's wall following behavior is quite different from that which has inspired many robotic researchers [13][18], [21], who focused on the classical honeybee's centering behavior observed by Srinivasan and Colleagues [3], [8], which they explained quite differently in terms of an 'optic flow balance' hypothesis. For the first time, the LORA robot controls its forward speed smoothly by jointly controlling the rear thrusters and the lift fan via a single controller (the forward controller). This control strategy enables the robot to speed up or slow down more efficiently. All the processing steps are carried out onboard thanks to the new generation of low-power $\mu C s$ obtained from Microchip(C). The LORA robot also carries its own power supplies (LiPo batteries) and the wireless communication link ("Bluetooth" module) used for data monitoring and higher order commands. The robot is therefore fully autonomous and moves freely without being encumbered by any umbilical link.

In conclusion, the LORA III autopilot is the first step toward a deft, lightweight (mass of the electronics: $8.17 \mathrm{~g}$ ), powerlean (power consumption: $0.75 \mathrm{~W}$ ) visuo-motor control system which could potentially be installed on a 3-D free-flying micro-air vehicle (MAV) by extending the visual field to include the ventral and dorsal parts, as simulated in [10]. This autopilot could also be applied to other types of (holonomic and fully actuated) vehicles such as blimps, autonomous underwater vehicles, and helicopters with counter-rotating rotors (in which pitch and roll are uncoupled). The next step will be to improve the OF-based autopilot by enlarging its FOV, and find an efficient means of controlling the heading (yaw $\Psi$ ) on a visual basis to enable the robot to successfully negotiate more challenging corridors comprising L-junctions or T-junctions.

\section{ACKNOWLEDGMENTS}

We thank Dr. J. Blanc for correcting the English manuscript, R. Brinkworth and D. O'Carroll (Adelaide University, Australia) for kindly making their High Dynamic Range panoramic images available to us, J. Diperi and Y. Luparini for their involvement in the mechanical design of the LORA robot and the test bench and M. Boyron for his involvement in the overall electronic design of the LORA robot. This research was supported partly by CNRS (Life Science; Information Science; Engineering Science and Technology) Aix-Marseille University and by the French National Research Agency (ANR) (EVA project under ANRContInt grant number ANR608-CORD-007-04).

\section{APPENDIX}

TABLE I

TRANSFER FUNCTIONS

\begin{tabular}{|c|c|}
\hline$H_{C}(s)=K_{c}$ & With $K_{c}=9.17 \cdot 10^{-3} \mathrm{~V} /{ }^{\circ}$ \\
\hline$H_{g}(s)=K_{g}$ & With $K_{g}=5 \cdot 10^{-3} \mathrm{~V} /\left(^{\circ} / s\right)$ \\
\hline$G_{\Psi}(s)=\frac{1}{s} \cdot \frac{H_{\Psi}}{1+\tau_{\Psi} \cdot s}$ & With $H_{\Psi}=4.4 \cdot 10^{-1} \mathrm{~V}$ \\
& and $\tau_{\Psi}=3 \cdot 10^{-1} \mathrm{~s}$ \\
\hline$G_{F w d}(s)=\frac{K_{F w d}}{1+\tau_{F w d} \cdot s}$ & See values in Table II \\
\hline$G_{\text {Side }}(s)=\frac{1}{s} \cdot \frac{K_{\text {Side }}}{1+\tau_{S i d e} \cdot s}$ & See values in Table III \\
\hline$C_{\Psi}(s)=K_{\Psi}$ & With $K_{\Psi}=3$ \\
\hline$C_{\Psi}(s)=K_{1} \cdot \frac{1+\tau_{1} \cdot s}{s}$ & With $K_{1}=5$ \\
& and $\tau_{1}=3 \cdot 10^{-1} \mathrm{~s}$ \\
\hline$C_{F w d}(s)=K_{2} \cdot \frac{1+\tau_{2} \cdot s}{s} \cdot \frac{1}{1+\tau_{3} \cdot s}$ & With $K_{2}=0.8$, \\
& $\tau_{2}=2.7 \cdot 10^{-1} \mathrm{~s}$ and \\
& $\tau_{3}=8 \cdot 10^{-3} \mathrm{~s}$ \\
\hline$C_{\text {Side }}(s)=K_{3} \cdot \frac{1+a \cdot T \cdot s}{1+T \cdot s} \cdot \frac{1}{1+\tau_{4} \cdot s}$ & With $K_{3}=4 \cdot 10^{-1}$, \\
& $a=5.7, T=2.1 \cdot 10^{-1}$ \\
& and $\tau_{4}=1.59 \cdot 10^{-2} \mathrm{~s}$ \\
\hline
\end{tabular}

TABLE II

FORWARD DYNAMICS PARAMETERS

\begin{tabular}{|c|c|c|c|}
\hline$u_{\text {LiftFan }}(\%)$ & $K_{F w d}\left(10^{-3} m \cdot s^{-1} \cdot \%^{-1}\right)$ & $\tau_{F w d}(s)$ & Fitfactor $(\%)$ \\
\hline 42 & 30.11 & 2.67 & 99.8 \\
\hline 41 & 28.11 & 2.64 & 99.3 \\
\hline 40 & 25.91 & 2.63 & 99.7 \\
\hline 39 & 20.88 & 2.02 & 99.4 \\
\hline 38 & 18.45 & 1.54 & 98.3 \\
\hline 37 & 16.29 & 1.34 & 96.1 \\
\hline
\end{tabular}

\section{REFERENCES}

[1] T. Collett, "Some operating rules for the optomotor system of a hoverfly during voluntary fligh," Journal of Comparative Physiology A, vol. 138, pp. 271-282, 1980.

[2] H. Wagner, "Flow-field variables trigger landing in flies," Nature, vol. 297, no. 5862, pp. 147-148, May 1982. 
TABLE III

SIDE DYNAMICS PARAMETERS

\begin{tabular}{|c|c|c|c|}
\hline$u_{\text {LiftFan }}(\%)$ & $K_{\text {Side }}\left(10^{-3} m \cdot s^{-1} \cdot \%^{-1}\right)$ & $\tau_{\text {Side }}(s)$ & Fitfactor $(\%)$ \\
\hline 42 & 16.28 & 2.54 & 94.32 \\
\hline 41 & 13.92 & 2.45 & 92.28 \\
\hline 40 & 11.02 & 2.2 & 92.11 \\
\hline 39 & 8.76 & 1.89 & 89.12 \\
\hline 38 & 8.32 & 1.48 & 88.72 \\
\hline 37 & 7.58 & 1.2 & 84.54 \\
\hline
\end{tabular}

[3] M. Srinivasan, M. Lehrer, W. Kirchner, and S. Zhang, "Range perception through apparent image speed in freely flying honeybees," Visual neuroscience, vol. 6(5), pp. 519-535, 1991.

[4] L. F. Tammero and M. H. Dickinson, "The influence of visual landscape on the free flight behavior of the fruit fly drosophila melanogaster," Journal of Experimental Biology, vol. 205(3), pp. 327343, 2002.

[5] J. Serres, G. Masson, F. Ruffier, and N. Franceschini, "A bee in the corridor: centering and wall-following," Naturwissenschaften, vol. 95, pp. 1181-1187, 2008.

[6] E. Baird, M. Srinivasan, S. Zhang, R. Lamont, and A. Cowling, "Visual control of flight speed and height in the honeybee," From Animals to Animats 9, pp. 40-51, 2006.

[7] G. Portelli, F. Ruffier, and N. Franceschini, "Honeybees change their height to restore their optic flow," Journal of Comparative Physiology A: Neuroethology, Sensory, Neural, and Behavioral Physiology, vol. 196(4), pp. 307-313, 2010.

[8] M. Srinivasan, S. Zhang, M. Lehrer, and T. Collett, "Honeybee navigation en route to the goal: visual flight control and odometry," Journal of Experimental Biology, vol. 199(1), pp. 237-44, 1996.

[9] E. Baird, T. Kornfeldt, and M. Dacke, "Minimum viewing angle for visually guided ground speed control in bumblebees," Journal of Experimental Biology, vol. 213(10), pp. 1625-1632, 2010.

[10] G. Portelli, F. L. Roubieu, F. Ruffier, and N. Franceschini, "Honeybees' speed depends on dorsal as well as lateral, ventral and frontal optic flows," PLoS ONE, vol. 6(5), p. e19486, 2011.

[11] J. M. Pichon, C. Blanes, and N. Franceschini, "Visual guidance of a mobile robot equipped with a network of self-motion sensors," unbekannt, vol. 1195, pp. 44-53, 1989.

[12] N. Franceschini, J. M. Pichon, and C. Blanes, "From insect vision to robot vision," Philosophical Transactions of the Royal Society B: Biological Sciences, vol. 337, pp. 283-294, 1992.

[13] D. Coombs and K. Roberts, "'Bee-bot': using peripheral optical flow to avoid obstacles," in Society of Photo-Optical Instrumentation Engineers (SPIE) Conference Series, vol. 1825, Boston, USA, September 1992, pp. 714-721.

[14] A. Duchon and W. Warren, "Robot navigation from a gibsonian viewpoint," in IEEE International Conference on Systems, Man, and Cybernetics, 1994.'Humans, Information and Technology'., vol. 3, San Antonio, USA, October 1994, pp. 2272-2277.

[15] J. Santos-Victor, G. Sandini, F. Curotto, and S. Garibaldi, "Divergent stereo in autonomous navigation: From bees to robots," International Journal of Computer Vision, vol. 14(2), pp. 159-177, 1995.

[16] K. Weber, S. Venkatesh, and M. Srinivasan, "Insect inspired behaviors for the autonomous control of mobile robots," in Proceedings of the 13th International Conference on Pattern Recognition, 1996., M. V. Srinivasan and S. Venkatesh, Eds. Vienna, Austria: U.K.: Oxford Univ. Press, August 1997, pp. 226-248.

[17] A. Argyros, D. Tsakiris, and C. Groyer, "Biomimetic centering behavior mobile robots with panoramic sensors," IEEE Robotics \& Automation Magazine, vol. 11(4), pp. 21-30, 2004.

[18] S. Hrabar, G. Sukhatme, P. Corke, K. Usher, and J. Roberts, "Combined optic-flow and stereo-based navigation of urban canyons for a UAV," in IEEE/RSJ International Conference on Intelligent Robots and Systems (IROS), Los Angeles, USA, August 2005, pp. 3309-3316.

[19] C. McCarthy, N. Barnes, and R. Mahony, "A robust docking strategy for a mobile robot using flow field divergence," IEEE Transactions on Robotics, vol. 24(4), pp. 832-842, 2008.

[20] J. Serres, D. Dray, F. Ruffier, and N. Franceschini, "A vision-based autopilot for a miniature air vehicle: joint speed control and lateral obstacle avoidance," Autonomous robot, vol. 25, pp. 103-122, 2008.
[21] J. Humbert and A. Hyslop, "Bioinspired visuomotor convergence," IEEE Transactions on Robotics, vol. 26(1), pp. 121-130, 2010.

[22] L. Muratet, S. Doncieux, Y. Briere, and J. Meyer, "A contribution to vision-based autonomous helicopter flight in urban environments,' Robotics and Autonomous Systems, vol. 50(4), pp. 195-209, 2005.

[23] F. Ruffier and N. Franceschini, "Optic flow regulation: the key to aircraft automatic guidance," Robotics and Autonomous Systems, vol. 50(4), pp. 177-194, 2005.

[24] J. Zufferey, A. Klaptocz, A. Beyeler, J. Nicoud, and D. Floreano, "A 10-gram vision-based flying robot," Advanced Robotics, vol. 21(14), pp. 1671-1684, 2007.

[25] A. Beyeler, J. Zufferey, and D. Floreano, "Vision-based control of near-obstacle flight," Autonomous Robots, vol. 27(3), pp. 201-219, 2009.

[26] F. Expert and F. Ruffier, "Controlling docking, altitude and speed in a circular high-roofed tunnel thanks to the optic flow," in IEEE/RSJ International Conference on Intelligent Robots and Systems (IROS), Vilamoura, Portugal, October 2012 (accepted).

[27] F. Ruffier, S. Viollet, S. Amic, and N. Franceschini, "Bio-inspired optical flow circuits for the visual guidance of micro-air vehicles," in IEEE International Symposium on Circuits and Systems (ISCAS), vol. 3, Bangkok, Thailand, May 2003, pp. 846-849.

[28] M. Pudas, S. Viollet, F. Ruffier, A. Kruusing, S. Amic, S. Leppävuori, and N. Franceschini, "A miniature bio-inspired optic flow sensor based on low temperature co-fired ceramics (LTCC) technology," Sensors and Actuators A: Physical, vol. 133(1), pp. 88-95, 2007.

[29] S. Viollet, F. Ruffier, T. Ray, M. Menouni, F. Aubépart, L. Kerhuel, and N. Franceschini, "Characteristics of three miniature bio-inspired optic flow sensors in natural environments," in Fourth International Conference on Sensor Technologies and Applications (SENSORCOMM), Venice, Italy, July 2010, pp. 51-55.

[30] F. Roubieu, F. Expert, M. Boyron, B.-J. Fuschlock, S. Viollet, and F. Ruffier, "A novel 1-gram insect based device measuring visual motion along 5 optical directions," in IEEE Sensors Conference, Limerick, Ireland, October 2011, pp. 687-690.

[31] F. Expert, S. Viollet, and F. Ruffier, "Outdoor field performances of insect-based visual motion sensors," Journal of Field Robotics, vol. 28(4), pp. 974-977, October 2011.

[32] L. Cremean, W. Dunbar, D. van Gogh, J. Hickey, E. Klavins, J. Meltzer, and R. Murray, "The Caltech Multi-Vehicle Wireless Testbed," in Proceedings of the 41st IEEE Conference on Decision and Control, vol. 1, Las Vegas, USA, December 2002, pp. 86-88.

[33] V. P. I Badia S. B., Bernardet U., "Non-linear neuronal responses as an emergent property of afferent networks: A case study of the locust lobula giant movement detector," PLoS Computational Biology, vol. 6(3), p. e1000701, 2010.

[34] S. Fuller and R. M. Murray, "A hovercraft robot that uses insectinspired visual autocorrelation for motion control in a corridor,' in IEEE International Conference on Robotics and Biomimetics (ROBIO), Karon Beach, Phuket, December 2011, pp. 1474-1481.

[35] H. Seguchi and T. Ohtsuka, "Nonlinear receding horizon control of an underactuated hovercraft," International Journal of Robust and Nonlinear Control, vol. 13(3-4), pp. 381-398, 2003.

[36] Z. Jin, S. Waydo, E. Wildanger, M. Lammers, H. Scholze, P. Foley, D. Held, and R. Murray, "MVWT-II: the second generation Caltech Multi-Vehicle Wireless Testbed," in Proceedings of the 2004 American Control Conference, vol. 6, Boston, USA, July 2004, pp. 53215326.

[37] J. Humbert, R. Murray, and M. Dickinson, "A control-oriented analysis of bio-inspired visuomotor convergence," in 44th IEEE Conference on Decision and Control and European Control Conference (CDC-ECC '05)., Seville, Spain, December 2005, pp. 245-250.

[38] J. Zufferey and D. Floreano, "Toward 30-gram autonomous indoor aircraft: Vision-based obstacle avoidance and altitude control," in Proceedings of the 2005 IEEE International Conference on Robotics and Automation (ICRA), Barcelona, Spain, April 2005, pp. 2594-2599.

[39] S. Viollet, L. Kerhuel, and N. Franceschini, "A 1-gram dual sensorless speed governor for micro-air vehicles," in 16th Mediterranean Conference on Control and Automation, Ajaccio, France, June 2008, pp. 1270-1275. 